\title{
Thromboembolic Complications in Patients with Clopidogrel Resistance after Coil Embolization for Unruptured Intracranial Aneurysms
}

\author{
B. Kim, K. Kim, P. Jeon, S. Kim, H. Kim, H. Byun, J. Cha, S. Hong, and K. Jo
}

\begin{abstract}
BACKGROUND AND PURPOSE: Antiplatelet resistance is known to be associated with symptomatic ischemic complication after endovascular coil embolization. The purpose of our study was to evaluate the relationship between antiplatelet resistance and clinically silent thromboembolic complications using DWI in patients who underwent coil embolization for unruptured intracranial aneurysm.
\end{abstract}

\begin{abstract}
MATERIALS AND METHODS: Between October 2011 and May 2013, 58 patients with 62 unruptured aneurysms who were measured for antiplatelet response using VerifyNow assay and underwent elective coil embolization for an unruptured aneurysm with posttreatment DWI were enrolled. Diffusion-positive lesions were classified into 3 groups according to the number of lesions $(n=0$ [grade 0 ], $n<6$ [grade I], and $n \geq 6$ [grade II]). The relationship between antiplatelet resistance and diffusion-positive lesions was analyzed.

RESULTS: Sixty-two endovascular coiling procedures were performed on 58 patients. Clopidogrel resistance was revealed in 23 patients (39.7\%) and diffusion-positive lesions were demonstrated in 28 patients (48.3\%); these consisted of 19 (32.8\%) grade I and 9 (15.5\%) grade II lesions. Clopidogrel resistance was not relevant to the development of any diffusion-positive lesion (grade I and II, $P=.789$ ) but was associated with the development of multiple diffusion-positive lesions (grade II, $P=.002$ ). In the logistic regression prediction model, clopidogrel resistance showed significant correlation with the development of grade II lesions $(P=.001)$.
\end{abstract}

CONCLUSIONS: Multiple diffusion-positive lesions ( $\geq 6$ in number) occurred more frequently in patients with clopidogrel resistance after endovascular coiling for unruptured aneurysms.

ABBREVIATION: PRU $=$ P2Y12 reaction units

S ince it was demonstrated that clopidogrel resistance is associated with thromboembolic events after cardiovascular stent placement, many studies in patients undergoing neurovascular stent placement have revealed a similar relationship between antiplatelet drug resistance and thromboembolic complications. ${ }^{1,2}$ Furthermore, recently published reports have documented that clopidogrel resistance had an effect on the occurrence of symptomatic ischemic infarcts in patients undergoing endovascular treatment with or without adjunctive devices for intracranial aneurysms. ${ }^{3,4}$

The widespread and routine application of DWI after coil em-

Received December 22, 2013; accepted after revision March 1, 2014

From the Departments of Radiology (B.K., K.K., P.J., S.K., H.K., H.B., J.C.) and Neurosurgery (S.H., K.J.), Samsung Medical Center, Sungkyunkwan University School of Medicine, Seoul, Korea.

Please address correspondence to Keonha Kim, MD, PhD, Department of Radiology, Samsung Medical Center, Sungkyunkwan University School of Medicine, 50, Irwon-dong, Gangnam-gu, Seoul, 135-710, Republic of Korea; e-mail: somatom. kim@samsung.com

http://dx.doi.org/10.3174/ajnr.A3955 bolization has revealed that clinically silent thromboembolic phenomena occur in up to one-third of cases. ${ }^{5}$ Several clinical and procedural factors have been proposed as being associated with those postprocedural diffusion abnormalities. ${ }^{6}$ However, the relationship between the antiplatelet drug reactivity and the thromboembolic complication delineated on DWI has not been wellcharacterized in patients undergoing coil embolization. The aim of the present study was to evaluate the frequency of posttreatment diffusion-positive lesions associated with coil insertion for treatment of unruptured intracranial aneurysms in patients with antiplatelet drug resistance. In addition, other clinical, aneurysmal, and procedural factors were assessed to reveal the association with the occurrence of posttreatment diffusion-positive lesions.

\section{MATERIALS AND METHODS \\ Patients}

From August 2011 to May 2013, a total of 328 patients with unruptured intracranial aneurysm were treated with elective endovascular coil embolization in our institute. During this period, patients who underwent coil embolization for unruptured aneu- 
Table 1: Patient characteristics, aneurysmal specifics, technical aspect of the procedure, and antiplatelet drug reactivity associated with any diffusion-positive lesion in patients undergoing endovascular coiling of unruptured intracranial aneurysms

\begin{tabular}{|c|c|c|c|c|c|}
\hline & Category $^{a}$ & $\begin{array}{l}\text { Grade } 0 \\
(n=30)\end{array}$ & $\begin{array}{c}\text { Grade I/II } \\
(n=28)\end{array}$ & $P_{\text {uni }}$ & $P_{\text {logistic }}$ \\
\hline \multirow[t]{2}{*}{ Sex } & Male $(23,39.7 \%)$ & 12 & 11 & .956 & \\
\hline & Female $(35,60.3 \%)$ & 18 & 17 & & \\
\hline \multirow[t]{2}{*}{ Age (years) } & $\leq 60(35,60.3 \%)$ & 21 & 14 & .179 & \\
\hline & $>60(23,39.7 \%)$ & 9 & 14 & & \\
\hline HTN & Yes $(23,39.7 \%)$ & 11 & 12 & .789 & \\
\hline DM & Yes $(4,6.9 \%)$ & 3 & 1 & .612 & \\
\hline Dyslipidemia & Yes (11, 19.0\%) & 4 & 7 & .325 & \\
\hline CAD & Yes $(3,5.2 \%)$ & 1 & 2 & .605 & \\
\hline Stroke & Yes $(6,10.3 \%)$ & 1 & 5 & .097 & .998 \\
\hline Statin & Yes (11, 19.0\%) & 5 & 6 & .744 & \\
\hline Aspirin & Yes $(19,32.8 \%)$ & 9 & 10 & .781 & \\
\hline Smoking & Yes $(15,25.9 \%)$ & 7 & 8 & .767 & \\
\hline \multirow[t]{2}{*}{ Dome size } & $<7$ mm $(39,67.2 \%)$ & 22 & 17 & .404 & \\
\hline & $\geq 7 \mathrm{~mm}(19,32.8 \%)$ & 8 & 11 & & \\
\hline \multirow[t]{2}{*}{ Neck width } & $<4 \mathrm{~mm}(32,55.2 \%)$ & 19 & 13 & .291 & \\
\hline & $\geq 4 \mathrm{~mm}(26,44.8 \%)$ & 11 & 15 & & \\
\hline \multirow[t]{2}{*}{ Multiplicity of the procedure ${ }^{b}$} & Single $(54,93.1 \%)$ & 30 & 24 & .048 & .072 \\
\hline & Double $(4,6.9 \%)$ & 0 & 4 & & \\
\hline \multirow[t]{3}{*}{ Procedural methods } & Single $(16,27.6 \%)^{c}$ & 10 & 6 & .453 & \\
\hline & Double $(18,41.4 \%)^{c}$ & 10 & 8 & & \\
\hline & Stent $(24,31 \%)$ & 10 & 14 & & \\
\hline Aspirin resistance & Yes $(9,15.5 \%)$ & 3 & 6 & .290 & .720 \\
\hline Clopidogrel resistance & Yes $(23,39.7 \%)$ & 11 & 12 & .789 & .998 \\
\hline
\end{tabular}

Note:- HTN indicates hypertension; DM, diabetes mellitus; CAD, coronary artery disease

${ }^{a}$ (number, percent).

b Multiple procedures in a single session.

c Single or double microcatheter technique, stent-assisted technique.

Table 2: Patient characteristics, aneurysmal specifics, technical aspect of the procedure, and antiplatelet drug reactivity associated with multiple diffusion-positive lesions in patients undergoing endovascular treatment of unruptured intracranial aneurysms

\begin{tabular}{|c|c|c|c|c|c|}
\hline & Category $^{a}$ & $\begin{array}{c}\text { Grade } 0 / 1 \\
(n=49)\end{array}$ & $\begin{array}{c}\text { Grade II } \\
(n=9)\end{array}$ & $P_{\text {uni }}$ & $P_{\text {logistic }}$ \\
\hline \multirow[t]{2}{*}{ Sex } & Male $(23,39.7 \%)$ & 20 & 3 & 1.000 & \\
\hline & Female $(35,60.3 \%)$ & 29 & 6 & & \\
\hline \multirow[t]{2}{*}{ Age (years) } & $\leq 60(35,60.3 \%)$ & 30 & 5 & 1.000 & \\
\hline & $>60(23,39.7 \%)$ & 19 & 4 & & \\
\hline HTN & Yes $(23,39.7 \%)$ & 20 & 3 & 1.000 & \\
\hline DM & Yes $(4,6.9 \%)$ & 4 & 0 & 1.000 & \\
\hline Dyslipidemia & Yes (11, 19.0\%) & 8 & 3 & .350 & \\
\hline CAD & Yes $(3,5.2 \%)$ & 2 & 1 & .403 & \\
\hline Stroke & Yes (6, 10.3\%) & 4 & 2 & .231 & \\
\hline Statin & Yes (11, 19.0\%) & 8 & 3 & .350 & \\
\hline Aspirin & Yes (19, 32.8\%) & 16 & 3 & 1.000 & \\
\hline Smoking & Yes $(15,25.9 \%)$ & 13 & 2 & 1.000 & \\
\hline \multirow{2}{*}{ Dome size } & $<7 \mathrm{~mm}(39,67.2 \%)$ & 34 & 5 & .456 & \\
\hline & $\geq 7 \mathrm{~mm}(19,32.8 \%)$ & 15 & 4 & & \\
\hline \multirow[t]{2}{*}{ Neck width } & $<4 \mathrm{~mm}(32,55.2 \%)$ & 28 & 4 & .717 & \\
\hline & $\geq 4 \mathrm{~mm}(26,44.8 \%)$ & 21 & 5 & & \\
\hline \multirow[t]{2}{*}{ Multiplicity of the procedure ${ }^{b}$} & Single $(54,93.1 \%)$ & 48 & 6 & .010 & .051 \\
\hline & Double $(4,6.9 \%)$ & 1 & 3 & & \\
\hline \multirow[t]{3}{*}{ Procedural methods } & Single $(16,27.6 \%)^{c}$ & 15 & 1 & .088 & .642 \\
\hline & Double $(18,41.4 \%)^{c}$ & 17 & 1 & & \\
\hline & Stent $(24,31 \%)$ & 17 & 7 & & \\
\hline Aspirin resistance & Yes $(9,15.5 \%)$ & 8 & 1 & 1.000 & .667 \\
\hline Clopidogrel resistance & Yes $(23,39.7 \%)$ & 15 & 8 & .002 & .014 \\
\hline
\end{tabular}

Note:- - HTN indicates hypertension; DM, diabetes mellitus; CAD, coronary artery disease.

${ }^{a}$ (number, percent).

${ }^{\mathrm{b}}$ Multiple procedures in a single session.

' Single or double microcatheter technique, stent-assisted technique.

rysm were prescribed dual antiplatelet therapy 7 days before the procedure and antiplatelet drug reactivity was measured 1 day before the procedure. In addition, posttreatment MR examina- tion including DWI was done within 48 hours after the procedure. Measurement of antiplatelet drug reactivity and posttreatment MR imaging, however, were performed on the basis of the clinician's individual policy. Therefore, we excluded 51 patients for whom posttreatment diffusion MR images were not available and 119 patients with an incomplete reactivity test for aspirin and clopidogrel. In addition, 100 patients were ruled out because of the lack of both preprocedural reactivity test and posttreatment DWI. Cases of ruptured aneurysm were not included to eliminate the effect of subarachnoid hemorrhage. Finally, we retrospectively reviewed 58 nonconsecutive patients with 62 unruptured intracranial aneurysms who underwent elective coil embolization from our prospective data base collected from August 2011 and May 2013.

The baseline clinical characteristics of the patients were reviewed via their medical charts in our institute and are presented in Tables 1 and 2. The clinical laboratory data of platelet activity and procedural information including specifics of the aneurysm, treatment methods, and devices used in the procedure and intraprocedural complications were recorded in our institute's prospectively maintained data base. The specifics of the aneurysm with which we were concerned were the width of the neck and the dome size of the sac, which were shown to be significantly associated with the rate of thromboembolic complications in the Analysis of Treatment by Endovascular approach of Non ruptured Aneurysms study. ${ }^{7}$ The treatment of 2 aneurysms in a single session was designated as multiple procedures.

\section{Medication Regimen and Platelet Function Testing}

Antiplatelet therapy was initiated 7 days before coiling procedure with the standard $100 \mathrm{mg}$ daily aspirin dose and $75 \mathrm{mg}$ daily clopidogrel dose. Because unplanned or unexpected coiling for ruptured aneurysm was already excluded from the present study, the regimen was uniformly adhered to by the enrolled patients. As a daily dose of clopidogrel, $75 \mathrm{mg}$ for 7 days was considered to be enough to reach the therapeutic range of antiplatelet effects, a loading dose of $300 \mathrm{mg}$ clopidogrel, which was the standard dosage for cardiovascular stent-placement procedures, was not adopted for our antiplatelet regimen. ${ }^{8,9}$ 
Response to the antiplatelet therapy was routinely checked 1 day before the procedure using a point-of-care antiplatelet function test (VerifyNow; Accumetrics, San Diego, California). The VerifyNow aspirin assay was employed to calculate the aspirin reaction units and the VerifyNow P2Y12 assay was utilized to obtain the $\mathrm{P} 2 \mathrm{Y} 12$ reaction units (PRU). Aspirin resistance was defined as aspirin reaction units $\geq 550$ following the manufacturer's suggestion and clopidogrel resistance was identified using a PRU $>240$ cutoff according to the results of a previous study. ${ }^{10,11}$ Patients were categorized into the resistant group if testing revealed the above defined levels after 7 days of dual antiplatelet therapy.

\section{Procedure and Procedure-Related Complications}

Endovascular coil embolization was performed following a standardized protocol in our neuroangiography suite under general anesthesia, as previously described. ${ }^{12}$ Systemic anticoagulation was achieved using an intravenous bolus injection of heparin (60 IU per $\mathrm{kg}, 3000 \sim 5000 \mathrm{IU}$, range) at the time of the guiding catheter insertion. An additional $1000 \mathrm{IU} / \mathrm{h}$ of heparin was administered to keep the activated coagulation time $2 \sim 3$-fold from the baseline with routine hourly check-ups. In the cases where a stent-assisted coil embolization technique was adopted, 24-hour heparinization was maintained after the procedure based on the
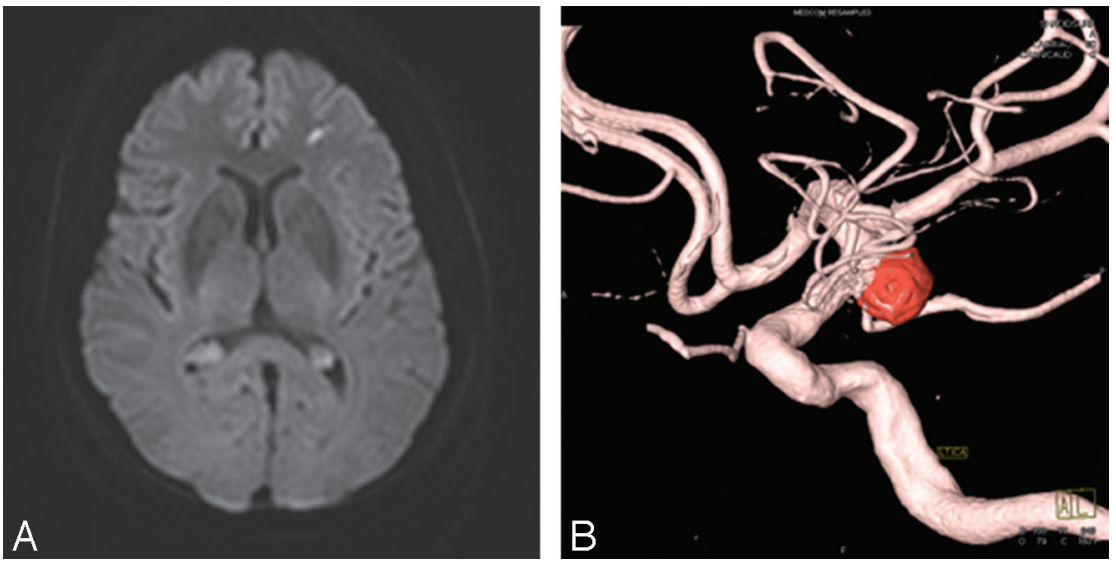

FIG 1. A 74-year-old female patient presented with left posterior communicating artery aneurysm and PRU of 134 (nonresistant group). A, Axial DWI demonstrates typical punctate diffusion restriction after an endovascular procedure. Furthermore, this lesion is related to clinically silent thromboembolic phenomena. $B, 3 D$ reconstruction image reveals coil embolized aneurysm arising from the origin of the posterior communicating artery.
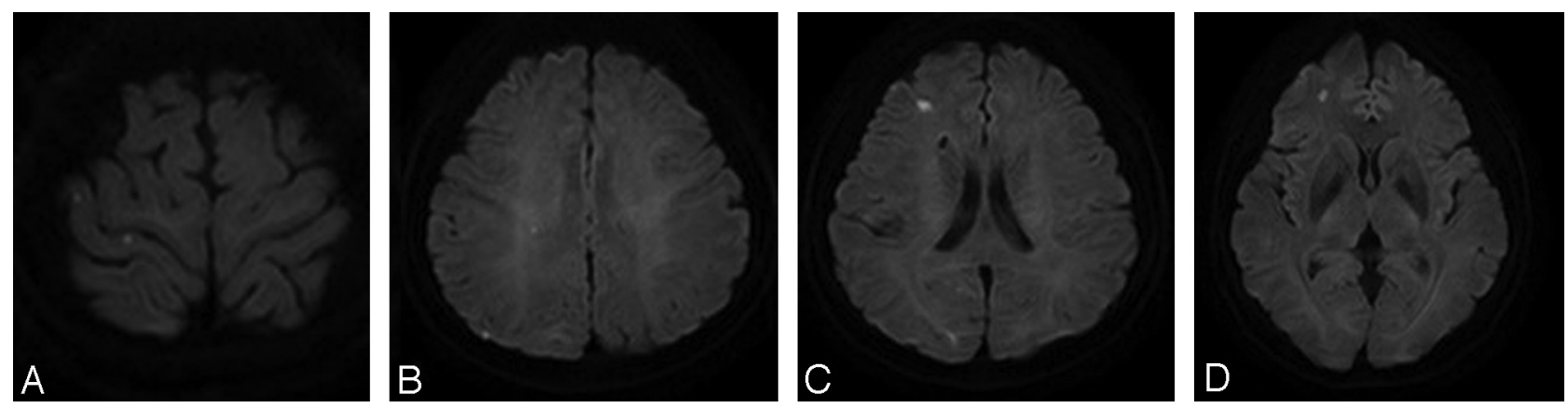

FIG 2. A 59-year-old female patient presented with right posterior communicating artery aneurysm and clopidogrel resistance detected with PRU of 304. A-D, Posttreatment DWI shows multiple scattered microembolic infarcts in right cerebral hemisphere, measured to be more than approximately 10 in number. 

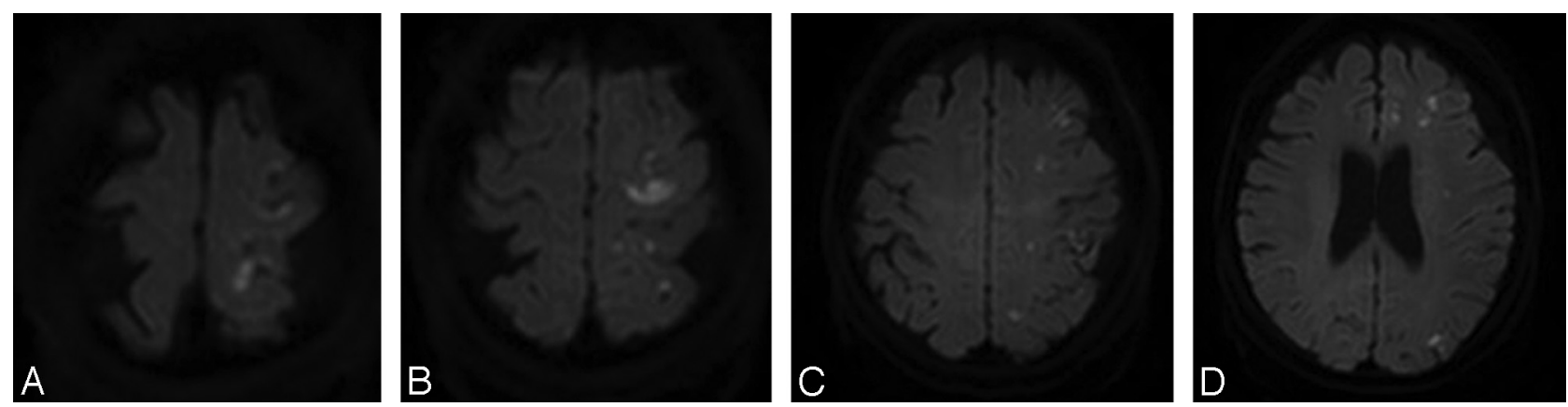

FIG 3. A 75-year-old female patient underwent stent-assisted coil embolization for unruptured left posterior communicating artery aneurysm The patient complained of dysarthria 22 hours after the procedure. On neurologic examination, abulia, unilateral homonymous hemianopsia, and transcortical sensory aphasia were noted. $A-D$, Posttreatment DWI shows numerous scattered diffusion-restrictive lesions in left cerebral hemisphere including larger lesion in left frontal cortex. PRU of the patient was 339.

relevance to the antiplatelet responsiveness were evaluated in the same manner.

\section{Statistical Analysis}

Statistical analysis was executed using SAS version 9.3 (SAS Institute, Cary, North Carolina). Univariable statistical analysis $\left(\chi^{2}\right.$ test or Fisher exact test) was performed to assess the associations between clinical, procedural, and laboratory variables and diffusion-positive lesion as a categoric variable. The variables with $P$ values $<.10$ in univariable analysis were chosen for multivariable models using multiple logistic regression analysis. Aspirin and clopidogrel resistance were included in the variables for multivariable analysis regardless of their $P$ values. The Firth penalized maximum likelihood estimation method was used in cases of rare events of variables. The same process of statistical analysis was conducted to evaluate multiple diffusion-positive lesions as a categoric variable. Statistical significance was defined as $P<.05$ in all analysis. In addition, $P$ values and $95 \%$ confidence intervals for odds ratio were corrected by the Bonferroni method because of multiple testing.

\section{RESULTS}

During the study period, overall 62 endovascular coiling procedures were performed on 58 patients, and 2 coil embolizations were undertaken in 4 patients in a single session. Among the 58 patients in whom platelet reactivity was measured, $15.5 \%(n=9)$ of the patients were aspirin-resistant and 39.7\% $(n=23)$ of the patients showed clopidogrel resistance. Five (8.6\%) patients were confirmed to be resistant to both aspirin and clopidogrel. In addition, the incidence of antiplatelet resistance in those who were excluded in this study because of the lack of posttreatment MR imaging $(n=51)$ was not appreciably different from that of the enrolled patients (aspirin resistance, 11.8\% $[n=6]$; clopidogrel resistance, $39.2 \%[n=20]$ ).

Intraprocedural adverse events occurred in $2(3.2 \%)$ of 62 cases, comprising 1/62 (1.6\%) intraprocedural thromboembolic events, and 1/62 (1.6\%) intraprocedural aneurysmal ruptures. The patient with intraprocedural thrombus formation in the parent artery was treated with an intra-arterial tirofiban infusion and several diffusion-positive lesions-less than 6 in number (grade I lesions)—were delineated on posttreatment DWI. No specific neurologic symptom was reported after the procedure. The pa- tient with the intraprocedural perforation was managed with further delivery of coils and the extravasation of contrast material was successfully controlled. However, intraprocedural thrombus formation developed during additional coil placement. Therefore, for a patient with aneurysmal leakage-induced thrombus formation, tirofiban was carefully administered after confirmation of the absence of extravasation of contrast material. Although the patient complained of headache after the procedure and multiple diffusion-positive lesions were demonstrated on postprocedural DWI (grade II lesions), neurologic evaluation revealed no neurologic deficit and the NIHSS was scored at 0 .

Postprocedural symptomatic ischemic complication without intraprocedural angiographic abnormality was reported in 1 (1.7\%) of the 58 patients. A 75 -year-old female patient with both aspirin and clopidogrel resistance presented with unruptured left posterior communicating artery aneurysm and endovascular treatment with stent-assisted coil embolization was performed. The patient complained of mild headache and dysarthria 22 hours after the procedure. On neurologic examination, abulia, unilateral homonymous hemianopsia, and transcortical sensory aphasia were detected and the NIHSS was scored at 4 (Fig 3). Multiple diffusion-restricted lesions, more than 6 in number, were delineated on posttreatment MR imaging as well. Prolonged heparinization and antiplatelet agent administration with the addition of ticlopidine $(500 \mathrm{mg} / \mathrm{d})$ were applied and the language problem resolved at discharge. No permanent neurologic deficit was reported and there was no procedure-related permanent morbidity or mortality.

Posttreatment MR imaging including DWI and MR angiography was obtained within 48 hours after the procedure in all 58 patients. Among them, 54 patients underwent posttreatment MR imaging as a routine protocol whereas 4 patients had a MR evaluation due to the presence of suspicious neurologic symptoms. Among these 4 patients, only 1 patient showed diffusion abnormalities (grade II lesions) and underwent MR evaluation 10 or 12 hours earlier than was expected because of development of aphasia. No diffusion abnormality was found in postprocedural MR imaging of the remaining 3 patients. The time interval between coil embolization and MR examination ranged from 23 to 46 hours (mean $=34.6$ hours). Diffusion-positive lesions on posttreatment DWI were present in $28(48.3 \%)$ of 58 patients, con- 
sisting of 19 (32.8\%) grade I and 9 (15.5\%) grade II lesions. All 28 patients showed diffusion-restricted lesions within the treated vascular territory and incidental diffusion abnormalities that occurred outside the treated vascular territory were found in 2 patients. As shown in Table 1, multiple procedures in a single session were higher in the diffusion-positive group (grade 0 versus I/II lesions, $P_{\text {uni }}=.048$ ). However, logistic regression analysis proved no significant difference in the occurrence of any diffusion-positive lesion $\left(P_{\text {logistic }}=.072\right.$ ). Other variables formerly known as association factors with thromboembolic complications such as age, dyslipidemia, prior thromboembolic event history, and the use of adjunctive devices did not show a statistical difference on the occurrence of any diffusion-positive lesion. In addition, neither aspirin nor clopidogrel resistance was proved to be associated with the occurrence of diffusion abnormality.

The incidence of multiple diffusion-positive lesions, more than 6 in number (grade II lesions), was higher in patients with multiple procedures in a single session and clopidogrel resistance on univariable analysis $\left(P_{\text {uni }}=.010\right.$ and $\left.P_{\text {uni }}=.002\right)$. However, only clopidogrel resistance showed significant association with the incidence of multiple diffusion-positive lesions in multivariable analysis $\left(P_{\text {logistic }}=.014\right.$, Table 2$)$. Furthermore, high signal intensities of grade II lesions showed larger size than those of grade I lesions and measured approximately $10-15 \mathrm{~mm}$ in diameter. These grade II lesions were grossly distinct from almost all grade I lesions by their larger size as well as irregular configuration (Fig 3).

\section{DISCUSSION}

Oral antiplatelet preparation before coiling of an unruptured cerebral aneurysm has been accepted as a standard protocol in many institutes nowadays since the publication of the first proposal by Workman et al. ${ }^{15}$ Moreover, there is a growing body of literature that describes the relationship between poor response to clopidogrel and postprocedural thromboembolic complications in patients undergoing endovascular treatment with stent assistance or the Pipeline Embolization Device (Covidien, Irvine, California). ${ }^{4,10,16}$ While the connection between clopidogrel resistance and intraprocedural thromboembolic complications or clinically recognized postprocedural ischemic deficits has been documented in prior studies, an association between antiplatelet resistance and silent ischemia as represented by diffusionweighted abnormalities has not been reported previously. In the present study, we focused on the frequency and number of diffusion-positive lesions according to the responsiveness to dual antiplatelet therapy with acetylsalicylic acid and clopidogrel. In addition, various clinical, aneurysmal, and technical factors were sought to find any correlation with diffusion-positive lesions.

Clinical implications of diffusion-positive lesions, known for clinically silent thromboembolic phenomena, are still controversial. First, there has been controversy whether silent ischemic lesions on DWI are really silent on clinical grounds. Many authors have raised the possibility of an association between diffusionpositive lesions and neuropsychological deficits with controversial results. ${ }^{17-19}$ It is documented that thorough neuropsychological examination can reveal significant cognitive impairments in patients with clinically silent diffusion-positive lesions on routine neurologic tests. Given the possible pathophysiological role of diffusion-positive lesions in cognitive decline, not only the frequency and but also the number of diffusion abnormalities were considered to be clinically relevant, even allowing for the contention that diffusion abnormalities do not necessarily translate into a clinically meaningful event. ${ }^{20,21}$ In general, the location of the lesion in an eloquent brain area is known to determine the development of neurologic deficits. However, a recently published report investigated the clinical relevance of the total number of diffusion-positive lesions and documented that the higher rate of diffusion-positive lesions can be a surrogate marker for symptomatic ischemia regardless of their location. ${ }^{22}$ Furthermore, they suggested the cutoff value of diffusion-positive lesions $(\geq 6)$ for predicting symptomatic ischemia. Therefore, the present study adopted the practice of categorizing patients into subgroups according to the number of diffusion-positive lesions (from grade 0 to III) based on those clinical implications of multiple microembolisms.

Several reports, including a pathologic study, suggested that the explainable embolic sources of diffusion-positive lesions after an endovascular procedure are air embolism, atheroma dislodged during catheterization, thrombus formation from the devices used over the course of the procedure, and hydrophilic coating from the catheters or wires. ${ }^{23-26}$ In this study, multiplicity of the procedure in a single session turned out to be associated with the occurrence of any diffusion-positive lesion. It was considered that multiple procedures in a single session had a tendency to increase the number of catheters used, the total procedure time, and the frequency of vessel probing, which was closely associated with mechanical stress. Therefore our results implied that the occurrence of diffusion-positive lesions was mainly contributed to by the factors that can exacerbate mechanical stress. Contrary to expectations, several factors that were documented to have an association with diffusion abnormalities such as dome diameter $\geq 7$ $\mathrm{mm}$, neck width $\geq 4 \mathrm{~mm}$, and patient age 60 years or older in prior studies had only a trend for the occurrence of diffusion-positive lesions and were not independent risk factors on univariate analysis. ${ }^{7,22,27}$

Although antiplatelet resistance had no relevance to occurrence of any diffusion abnormality, clopidogrel resistance showed significant association with the development of multiple diffusion-positive lesions. Among 9 patients with multiple diffusionpositive lesions (grade II lesions), 8 patients ( $n=8,88.9 \%$ ) showed clopidogrel resistance, which was significantly higher than the risk in patients who did not exhibit clopidogrel resistance $(n=1,11.1 \%)$. Moreover, 5 patients with multiple diffusionpositive lesions and clopidogrel resistance displayed numerous (more than 10 in number) tiny diffusion-restricted lesions in the corresponding territory of parent and distal cerebral arteries of the aneurysm. It was therefore speculated that active thrombus formation from endovascular devices by poor platelet inhibition seemed to mainly contribute to the formation of those multiple scattered embolic infarcts. Given the fact that 1 patient with a symptomatic ischemic event was classified as having grade II lesions, clopidogrel resistance seemed to be associated with clini- 
cally relevant diffusion-positive lesions in patients undergoing elective coil embolization for unruptured aneurysms.

Use of adjunctive devices for treatment of aneurysms with an unfavorable configuration for conventional coil embolization was proposed as a risk factor that can increase the incidence of thromboembolic complications compared with the single microcatheter technique by some authors, including the author of the prior study from our institute. ${ }^{12}$ However, other authors have published studies that show adjunctive device usage does not appear to increase the frequency of embolic or ischemic events. ${ }^{13}$ Alternatively, it was posed that the difference in preoperative antiplatelet medical management in each procedure could be relevant to the occurrence of thromboembolic complications. Our results showed that a difference in procedural devices such as the use of a stent or 2 microcatheters did not influence the development of diffusion-positive lesions under the same premedication strategy. As concluded in the prior study from our institute, appropriate and uniform premedication in a homogeneous patient group and adequate heparinization in all procedures may preclude the difference in the development of thromboembolic complications according to adjunctive devices.

There are several limitations to the present study. The retrospective nature of this study is the first limitation. Second, many patients were excluded for the lack of preprocedural drug reactivity test and postprocedural MR images according to the clinician's individual preference. One attending clinician routinely checked postprocedural MR examination within 1 or 2 postoperative days, whereas the remaining 2 clinicians checked MR imaging in symptomatic cases only. This selection bias might contribute to a higher thromboembolic complication rate. Third, there are no preprocedural MR images for comparison. Given some evidence that aneurysms alone can cause thromboembolic phenomena, this limitation can increase the frequency of diffusion-positive lesions artificially. ${ }^{28}$ In addition, we excluded patients with ruptured aneurysms for the reason that SAH may also cause diffusion-weighted imaging changes, but an intraprocedural rupture case with subsequent SAH was included. Fourth, a thorough clinical correlation with diffusion-positive lesions was not performed. Although NIHSS was performed on all patients after the procedure, a detailed neuropsychological examination that might help to delineate the clinical implications of diffusion-positive lesions was not performed. Lastly, there was no consideration regarding the degree of atherosclerosis in the aortic arch.

\section{CONCLUSIONS}

Antiplatelet drug resistance does not appear to be related with the occurrence of any diffusion-positive lesion after an endovascular coiling procedure for unruptured aneurysm. However, it seems that clopidogrel resistance is associated with the occurrence of multiple diffusion-positive lesions, especially those that are more than 6 in number. The present study suggests that giving premedication consisting of aspirin and clopidogrel and the measurement of antiplatelet activity should be done for patients scheduled to undertake elective coil embolization for unruptured aneurysms. In cases with clopidogrel resistance, adequate modification of the premedication may help to reduce the development of both clinically silent and evident thromboembolic complications after the procedure, and further study should be performed to seek the proper modification of the premedication.

\section{REFERENCES}

1. Gurbel PA, Bliden KP, Hiatt BL, et al. Clopidogrel for coronary stenting: response variability, drug resistance, and the effect of pretreatment platelet reactivity. Circulation 2003;107:2908-13

2. Fifi JT, Brockington C, Narang J, et al. Clopidogrel resistance is associated with thromboembolic complications in patients undergoing neurovascular stenting. AJNR Am J Neuroradiol 2013;34:716-20

3. Kang HS, Kwon BJ, Kim JE, et al. Preinterventional clopidogrel response variability for coil embolization of intracranial aneurysms: clinical implications. AJNR Am J Neuroradiol 2010;31:1206-10

4. Delgado Almandoz JE, Kadkhodayan Y, Crandall BM, et al. Variability in initial response to standard clopidogrel therapy, delayed conversion to clopidogrel hyper-response, and associated thromboembolic and hemorrhagic complications in patients undergoing endovascular treatment of unruptured cerebral aneurysms. J Neurointerv Surg 2014 Jan 7. [Epub ahead of print]

5. Cronqvist M, Wirestam R, Ramgren B, et al. Diffusion and perfusion MRI in patients with ruptured and unruptured intracranial aneurysms treated by endovascular coiling: complications, procedural results, MR findings and clinical outcome. Neuroradiology 2005; 47:855-73

6. Altay T, Kang HI, Woo HH, et al. Thromboembolic events associated with endovascular treatment of cerebral aneurysms. J Neurointerv Surg 2011;3:147-50

7. Pierot L, Spelle L, Vitry F, et al. Immediate clinical outcome of patients harboring unruptured intracranial aneurysms treated by endovascular approach: results of the ATENA study. Stroke 2008;39:2497-504

8. Lee DH, Arat A, Morsi H, et al. Dual antiplatelet therapy monitoring for neurointerventional procedures using a point-of-care platelet function test: a single-center experience. AJNR Am J Neuroradiol 2008;29:1389-94

9. Gurbel PA, Tantry US. Clopidogrel resistance? Thromb Res 2007;120:311-21

10. Delgado Almandoz JE, Crandall BM, Scholz JM, et al. Pre-procedure P2Y12 reaction units value predicts perioperative thromboembolic and hemorrhagic complications in patients with cerebral aneurysms treated with the Pipeline embolization device. J Neurointerv Surg 2013; 5(suppl 3):iii3-10

11. Maruyama H, Takeda H, Dembo T, et al. Clopidogrel resistance and the effect of combination cilostazol in patients with ischemic stroke or carotid artery stenting using the VerifyNow P2Y12 assay. Intern Med 2011;50:695-98

12. Jo KI, Yeon JY, Kim KH, et al. Predictors of thromboembolism during coil embolization in patients with unruptured intracranial aneurysm. Acta Neurochir (Wien) 2013;155:1101-06

13. Brooks NP, Turk AS, Niemann DB, et al. Frequency of thromboembolic events associated with endovascular aneurysm treatment: retrospective case series. J Neurosurg 2008;108:1095-100

14. Adams HP Jr, Bendixen BH, Kappelle LJ, et al. Classification of subtype of acute ischemic stroke. Definitions for use in a multicenter clinical trial. TOAST-Trial of Org 10172 in acute stroke treatment. Stroke 1993;24:35-41

15. Workman MJ, Cloft HJ, Tong FC, et al. Thrombus formation at the neck of cerebral aneurysms during treatment with Guglielmi detachable coils. AJNR Am J Neuroradiol 2002;23:1568-76

16. Delgado AlmandozJE, Crandall BM, Scholz JM, et al. Last-recorded P2Y12 reaction units value is strongly associated with thromboembolic and hemorrhagic complications occurring up to 6 months after treatment in patients with cerebral aneurysms treated with the Pipeline embolization device. AJNR Am J Neuroradiol 2014;35:128-35

17. Bendszus M, Stoll G. Silent cerebral ischaemia: hidden fingerprints of invasive medical procedures. Lancet Neurol 2006;5:364-72 
18. Knipp SC, Matatko N, Wilhelm H, et al. Evaluation of brain injury after coronary artery bypass grafting. A prospective study using neuropsychological assessment and diffusion-weighted magnetic resonance imaging. Eur J Cardiothorac Surg 2004;25:791-800

19. Restrepo L, Wityk RJ, Grega MA, et al. Diffusion- and perfusionweighted magnetic resonance imaging of the brain before and after coronary artery bypass grafting surgery. Stroke 2002;33:2909-15

20. Rordorf G, Bellon RJ, Budzik RE, Jr., et al. Silent thromboembolic events associated with the treatment of unruptured cerebral aneurysms by use of Guglielmi detachable coils: prospective study applying diffusion-weighted imaging. AJNR Am J Neuroradiol 2001;22:5-10

21. van Rooij WJ, Sluzewski M, Beute GN, et al. Procedural complications of coiling of ruptured intracranial aneurysms: incidence and risk factors in a consecutive series of 681 patients. AJNR Am J Neuroradiol 2006;27:1498-501

22. Kang DH, Kim BM, Kim DJ, et al. MR-DWI-positive lesions and symptomatic ischemic complications after coiling of unruptured intracranial aneurysms. Stroke 2013;44:789-91

23. Hu YC, Deshmukh VR, Albuquerque FC, et al. Histopathological assessment of fatal ipsilateral intraparenchymal hemorrhages after the treatment of supraclinoid aneurysms with the Pipeline embolization device. J Neurosurg 2014;120:365-74

24. Kim BJ, Lee SW, Park SW, et al. Insufficient platelet inhibition is related to silent embolic cerebral infarctions after coronary angiography. Stroke 2012;43:727-32

25. Busing KA, Schulte-Sasse C, Fluchter S, et al. Cerebral infarction: incidence and risk factors after diagnostic and interventional cardiac catheterization-prospective evaluation at diffusion-weighted MR imaging. Radiology 2005;235:177-83

26. Brockmann C, Hoefer T, Diepers M, et al. Abciximab does not prevent ischemic lesions related to cerebral angiography: a randomized placebo-controlled trial. Cerebrovasc Dis 2011;31:353-57

27. Albayram S, Selcuk H, Kara B, et al. Thromboembolic events associated with balloon-assisted coil embolization: evaluation with diffusion-weighted MR imaging. AJNR Am J Neuroradiol 2004; 25:1768-77

28. Qureshi AI, Mohammad Y, Yahia AM, et al. Ischemic events associated with unruptured intracranial aneurysms: multicenter clinical study and review of the literature. Neurosurgery 2000;46:282-89; discussion 89-90 